Received: 26.07 .2019

Revised: 17.08.2019

Accepted: 30.08 .2019

DOI: $10.17804 / 2410-9908.2019 .4 .006-015$

\title{
ON THE SEARCH OF NEW MAGNETIC MATERIALS FOR CRYOGENICS
}

\author{
E. Z. Valiev ${ }^{\text {a)* }}$ and V. I. Bobrovskii ${ }^{\text {b) }}$ \\ M.N. Miheev Institute of Metal Physics, Ural Branch of the Russian Academy of Sciences, \\ 18 S. Kovalevskoy St., Ekaterinburg, 620108, Russian Federation \\ a) (iD https://orcid.org/0000-0002-0760-0370 valiev@imp.uran.ru; \\ b) iD https://orcid.org/0000-0002-4692-8889 bobrovskii@imp.uran.ru \\ *Corresponding author. E-mail: valiev@imp.uran.ru \\ Address for correspondence: ul. S. Kovalevskoy, 18, 620108, Ekaterinburg, Russian Federation \\ Tel.: +7 (343) 3740003
}

Due to the latest achievements and potential practical applicability, magnetic refrigeration is currently of great interest. For the search of new effective materials for refrigerators with magnetic cooling, we obtain and analyze the governing equations enabling one to calculate the basic characteristics of the magnetocaloric effect (MCE) in magnetically ordered materials (temperature and entropy changes under on/off switching of the magnetic field). Recommendations and demands for the characteristics of promising ferro- and ferrimagnets in search are proposed on the basis of a joint analysis of these formula and experimental results.

Keywords: magnetic refrigeration, ferromagnets, antiferromagnets, ferrimagnets, magnetic phase transitions, magnetocaloric effect.

\section{Acknowledgment}

The work was performed with the use of IMP Neutron Material Science Complex within the state assignment for IPM UB RAS (theme Flux) and supported by the Basic Research Program of UB RAS, grant No. 18-10-2-22.

\section{References}

1 Franco V., Blázquez J.S., Ipus J.J., Law J.Y., Moreno-Ramírez L.M., Conde A. Magnetocaloric effect: From materials research to refrigeration device. Progress in Materials Science, 2018, vol. 93, pp. 112-232. DOI: 10.1016/j.pmatsci.2017.10.005.

2 Zimm C., Jastrab A., Sternberg A., Pecharsky V., Gschneidner K., Osborne M., Anderson I. Description and performance of a near-room temperature magnetic refrigerator. In: Kittel P., ed. Advances in Cryogenic Engineering Book Series (ACRE, vol. 43), Springer, Boston, MA, 1998, pp. 1759-1766. DOI: 10.1007/978-1-4757-9047-4_222. Online ISBN: 978-1-4757-9047-4.

3 Bean C.P., Rotbell D.C. Magnetic disorder as first-order phase transition. Phys. Rev., 1962, vol. 126, pp. 104-115. DOI: 10.1103/PhysRev.126.104.

4 Kittel C. Model of exchange-inversion magnetization. Phys. Rev., 1960, vol. 120, pp. 335-342. DOI: 10.1103/PhysRev.120.335.

$5 \quad$ Valiev E.Z., Kazantsev V. A. Magnetocaloric effect in $\mathrm{La}\left(\mathrm{Fe}_{x} \mathrm{Si}_{1-x}\right)_{13}$ ferromagnets. Physics of Metals and Metallography, 2011, vol. 113, no. 6, pp. 1000-1005. DOI: 10.1134/S1063776111150118.

6 Valiev E.Z. Isotropic Magnetoelastic Interaction in Two-Sublattice Ferri- and Antiferromagnets: Mean-Field Approximation for the Heisenberg Model. Physics of Metals and Metallography, 2003, vol. 96, no. 2, pp.121-127. 
7 Valiev E.Z. Entropy and Magnetocaloric Effect in Ferrimagnets $\mathrm{RCo}_{2}$. Physics of Metals and Metallography, 2017, vol. 124, no. 6, pp. 968-974. DOI: 10.1134/S1063776117060048.

8 Valiev E., Gimaev R., Zverev V., Kamilov K., Pyatakov A., Kovalev B., Tishin A. Application of the exchange-striction model for the calculation of the FeRh alloys magnetic properties. Intermetallics, 2019, vol. 108, pp. 81-86. DOI: 10.1016/j.intermet.2019.02.015.

9 Valiev E.Z. Entropy and Magnetocaloric Effect in Ferromagnets and Antiferromagnets. Physics of Metals and Metallography, 2007, vol. 104, no. 1, pp. 8-12. DOI: 10.1134/S0031918X07070022.

10 Valiev E.Z. Entropy and magnetocaloric effects in ferromagnets undergoing firstand second-order magnetic phase transitions. JETP, 2009, vol. 108, no. 2, pp. 279-285. DOI: $10.1134 / \mathrm{S} 1063776109020101$.

11 Fujita A., Akamatsu Y., Fukamishi K. Itinerant-electron metamagnetic transition in $\mathrm{La}\left(\mathrm{Fe}_{\mathrm{x}} \mathrm{Si}_{1-\mathrm{x}}\right)_{13}$ intermetallic compounds. J. Appl. Phys., 1999, vol. 85, pp. 4756-4758. DOI: 10.1063/1.370471.

12 Fujita A., Fujieda S., Hasegava Y., Fukamishi K. Itinerant-electron metamagnetic transition and large magnetocaloric effect in $\mathrm{La}\left(\mathrm{Fe}_{\mathrm{x}} \mathrm{Si}_{1-\mathrm{x}}\right)_{13}$ compounds and their hydrides. Phys. Rev. B, 2003, vol. 67, no 10, pp. 104416. DOI:10.1103/PhysRevB.67.104416. 
Подана в журнал: 26.07.2019

УДК 537.622.6:537.634.2

DOI: $10.17804 / 2410-9908.2019 .4 .006-015$

\title{
ПОИСК НОВЫХ МАГНИТНЫХ МАТЕРИАЛОВ ДЛЯ КРИОГЕННОЙ ТЕХНИКИ
}

\author{
Э. 3. Валиев ${ }^{\text {a)* }}$ В. И. Бобровский ${ }^{\text {) }}$ \\ ФГБУН Институт физики металлов имени М.Н. Михеева Уральского отделения Российской академии наук, \\ ул. С. Ковалевской, 18, г. Екатеринбург, Российская Федеращия \\ a) iD https://orcid.org/0000-0002-0760-0370 valiev@imp.uran.ru; \\ б) iD https://orcid.org/0000-0002-4692-8889 bobrovskii@imp.uran.ru \\ *Ответственный автор. Электронная почта: valiev@imp.uran.ru \\ Адрес для переписки: ул. С. Ковалевской, 18, 620108, г. Екатеринбург, Российская Федерация \\ Тел.: +7 (343) 374-00-03
}

Благодаря последним достижениям и потенциальным возможностям практического применения в настоящее время возник большой интерес к магнитной криогенике. Для поиска новых эффективных материалов для систем магнитного охлаждения получены формулы, позволяющие рассчитывать основные характеристики магнитокалорического эффекта (МКЭ) в магнитоупорядоченных веществах (адиабатическое изменение температуры и изотермическое изменение энтропии магнетика, при включении и выключении магнитного поля). На основе анализа этих теоретических выражений и накопленных экспериментальных данных предложены рекомендации и требования, которым должны удовлетворять перспективные ферро- и ферримагнетики для того, чтобы их характеристики МКЭ принимали максимальные значения.

Ключевые слова: магнитное охлаждение, ферромагнетики,антиферромагнетики, ферримагнетики, магнитные фазовые переходы, магнитокалорический эффект.

\section{1. Введение}

В обеспечении функционирования современного общества большую роль играет производство холода, основанное на использовании рефрижераторов различного типа, являющееся весьма энергозатратным и часто не вполне экологически безопасным в силу использования в этих машинах специфических хладоагентов. Поэтому большой интерес вызывают альтернативные методы получения низких температур. Уже более 100 лет известен магнитокалорический эффект (МКЭ), проявляющийся в изменении температуры вещества при изменении приложенного магнитного поля. Весьма ярко он проявляется в условиях тепловой изоляции материалов при выключении (или включении) внешнего магнитного поля, влекущем за собой перестройку состояния магнитной подсистемы. Наиболее универсальным мерилом таких перестроек является величина изменения энтропии этой подсистемы. В адиабатических условиях сохранения полной энтропии материала это означает соответствующее изменение (только с противоположным знаком) энтропии кристаллической решетки и, как следствие, температуры:

$$
\Delta T=-\frac{T}{C_{P, H}} \Delta S_{M}
$$

где $C_{P, H}$ и $\Delta S_{M}$ - теплоемкость материала и изменение энтропии магнитной подсистемы соответственно. 
МКЭ наблюдается в различных магнитных материалах (парамагнетиках, феррои ферримагнетиках) и уже достаточно давно используется в экзотических устройствах для получения сверхнизких температур. Но в последние годы прогресс в теоретическом и экспериментальном исследовании магнитотепловых свойств материалов вызвал новый интерес к МКЭ. Так, впервые удалось сконструировать магнитный холодильник, работоспособный при комнатной температуры [1]. В настоящее время технология производства магнитных рефрижераторов стремительно развивается.

Однако соединения со значительными величинами МКЭ не слишком многочисленны. Соответственно большой интерес представляет разработка методик их осознанного поиска или синтеза. Обычно при определении характеристик МКЭ (адиабатического изменения температуры $\Delta T_{a d}$ и изотермического изменения энтропии $\Delta S_{M}$ ) используют общие термодинамические соотношения $[1,2]$ и экспериментальные данные. При этом не удается выяснить зависимость характеристик МКЭ от ряда физических величин. Особенно это касается большого (гигантского), $\Delta T_{a d} \approx 10 \mathrm{~K} ; \Delta S_{M} \approx 30 \mathrm{~J} / \mathrm{kg} \cdot \mathrm{K}$ ) МКЭ, проявляющегося в некоторых материалах. Для решения этой проблемы необходимо привлечение продвинутых моделей описания магнетиков.

Мы покажем, что несмотря на существенные отличия всех этих систем для описания их термодинамических свойств можно предложить универсальный феноменологический подход, являющийся обобщением теорий Бина-Ротбелла [3] и Киттеля [4]. При этом важными элементами усовершенствования теории является включение в рассмотрение эффектов теплового расширения решетки. Будут получены термодинамические соотношения для максимальных значений $\Delta T_{a d}$ и $\Delta S_{M}$ и проведено их обсуждение и сравнение с экспериментом для ферромагнетиков (железо, никель гадолиний) с магнитными фазовыми переходами II рода, феррои ферримагнетиков $\mathrm{La}(\mathrm{FeSi})_{13}$ и $\mathrm{RCo}_{2}$, испытывающих магнитный фазовый переход I рода, и для отличающегося рекордными характеристиками МКЭ эквиатомного сплава Fe-Rh, в котором происходит переход I рода между ферромагнитным и антиферромагнитным состояниями. В заключение приведены требования, каким должны удовлетворять ферромагнетики для того чтобы магнитотепловые эффекты в них были максимальными.

\section{2. Постановка задачи и методы решения}

На примере ферромагнетика с одним сортом магнитных атомов, руководствуясь основными идеями обменно-стрикционной модели $[4,5]$, получим выражения для основных характеристик МКЭ. Обсуждение особенностей МКЭ в антиферромагнетиках и ферримагнетиках для конкретных соединений можно найти в наших работах [6-8].

Выражение для термодинамического потенциала (свободной энергии) ферромагнетика запишем в виде [5]:

$$
F=N J s^{2} m^{2}-N k_{B} T \ln Z(x)+\frac{1}{2} K^{-1} \omega^{2}+P \omega+N k_{B} T\left[3 \ln \left(1-\exp \left(-\frac{\Theta}{T}\right)\right)-D\left(\frac{\Theta}{T}\right)\right]
$$

где введены обозначения:

$$
\left\{\begin{array}{c}
x=\frac{2 \mu_{B} s H+2 s^{2} J m}{k_{B} T} \\
J=J_{0}+\gamma \omega \\
Z(x)=\frac{\operatorname{sh}\left(x+\frac{x}{2 s}\right)}{\operatorname{sh}\left(\frac{x}{2 s}\right)} \\
\Theta=\Theta_{0}+\Gamma \omega
\end{array}\right.
$$


Здесь $N$ - число магнитных атомов в единице объема; $J$ - обменный интеграл между выделенным атомом и всеми атомами его окружения с учетом в линейном приближении сжимаемости решетки; $\mu_{B}$ - магнетон Бора; $s$ - спин атома; $H$ - внешнее магнитное поле; $k_{B}-$ постоянная Больцмана; $\gamma$ - постоянная обменно-упругого взаимодействия (магнитная константа Грюнайзена); $K$ - сжимаемость; $P$ - давление; $m$ - приведенная намагниченность; $\omega=\Delta V / V-$ относительное изменение объема; $\Theta$ - температура Дебая; $Г$ - постоянная Грюнайзена; $D(\Theta / T)]$ - функция Дебая.

В уравнении (2) первые два слагаемых представляют вклад обменного взаимодействия в приближении молекулярного поля $[6,7]$, третье и четвертое слагаемые - вклад энергии объемных деформаций. Пятое слагаемое - вклад решетки в приближении Дебая Грюнайзена. Основная идея теорий Бина-Родбелла и Киттеля сводилась к учету зависимости обменного интеграла от объема (в частности, от теплового расширения решетки). В выражении для термодинамического потенциала (2) мы дополнили ее учетом зависимости температуры

Дебая от объема.

Из условия минимума свободной энергии по $m$ и $\omega$ следуют равновесные уравнения состояния для магнитной подсистемы и решетки:

$$
\left\{\begin{array}{c}
m=B_{S}(x) \\
\omega=\left(N \gamma s^{2} m^{2}+3 N k_{B} \frac{T}{\Theta} \Gamma \cdot D\left(\frac{\Theta}{T}\right)-P\right) K^{\prime}
\end{array}\right.
$$

Здесь $B_{s}(x)$ - функция Бриллюэна для спина $s ; \omega$ при $P=0$ есть спонтанная объемная магнитострикция плюс обычное тепловое расширение решетки. Отметим, что второе слагаемое в выражении (4) для $\omega$ оказывается важным только при обсуждении причины фазового перехода антиферромагнетик - ферромагнетик в сплаве $\mathrm{Fe}-\mathrm{Rh}$ [8]. В остальных случаях им можно пренебречь.

Для магнитной энтропии из $(2,4)$ следует

$$
S_{M}=N k_{B}[\ln Z(x)-m x] .
$$

Согласно экспериментам максимальные значения МКЭ наблюдаются в точке Кюри, что позволяет при вычислении изменения энтропии в магнитном поле использовать выражение (5) в приближении $x<<1$. В результате

$$
\Delta S_{M}^{\max }=S_{M}(H)-S_{M}(0)=-N k_{B} \frac{3 s}{2 s+2} m^{2} .
$$

Как следует из (4) и (5), зависимость энтропии от внешних условий определяется величиной намагниченности. Для адиабатического изменения температуры тогда немедленно получаем:

$$
\Delta T_{a d}^{\max } \approx-\frac{\Delta S_{M}^{\max } T_{C}}{C}=\frac{1}{C} N k_{B} T_{C} m^{2} \frac{3 s}{2 s+2}
$$

где $C$ - теплоемкость, а $T_{C}-$ температура Кюри.

\section{3. Результаты и обсуждение}

Формулы (1)-(7) позволяют проводить расчет термодинамических свойств ферромагнетиков с магнитными фазовыми переходами I и II рода, в зависимости от величины константы магнитоупругого взаимодействия $\gamma$. Если величина постоянной $\gamma$ меньше критического значения $\gamma_{C R}$, то переход будет фазовым переходом II рода. Если же $\gamma>\gamma_{C R}$, то возникает 
магнитный фазовый переход I рода [8]. Изменение рода фазового перехода происходит в трикритической точке при $\gamma=\gamma_{C R}$. Выражение для $\gamma_{C R}$ можно найти в работе [6].

Для $T=T_{C}$ в области магнитного фазового перехода II рода (т. е. $\gamma \ll \gamma_{C R}$ ) легко получить с использованием (4) и (6):

$$
\Delta S_{M}^{\max } \approx\left(2 \mu_{B} s H / k_{B} T_{c}\right)^{2 / 3}
$$

То есть в магнитном поле максимальное значение изменения энтропии (5) определяется отношением зеемановской и обменной энергий в степени $2 / 3$. Из формулы (8) следует, что наибольшие значения $\Delta S_{M}^{\max }$ и $\Delta T_{a d}^{\max }$ следует ожидать в ферромагнетиках с большими магнитными моментами и малыми значениями температуры Кюри. Этот вывод подтверждается расчетом и экспериментальными данными для никеля $\left(s=1 / 2, T_{C}=631 \mathrm{~K}\right)$, железа $(s=1$, $\left.T_{C}=1043 \mathrm{~K}\right)$ и гадолиния $\left(s=7 / 2, T_{C}=293 \mathrm{~K}\right)$. В [9] показано, что $\Delta S_{M}^{\max }$ и $\Delta T_{a d}^{\max }$ для гадолиния в 3-5 раз больше, чем для никеля и железа (для гадолиния $\Delta S_{M}^{\max } \approx 15 \mathrm{~J} / \mathrm{kg} \mathrm{K} ; \Delta T_{a d}^{\max } \approx 10 \mathrm{~K}$ при $H=5$ Т [9]).

Весьма интересные результаты можно получить, если проследить, как изменяются величины $\Delta S_{M}^{\max }$ и $\Delta T_{a d}^{\max }$ с ростом постоянной $\gamma$ при изменении рода магнитного фазового перехода со II на I, которое происходит в трикритической точке, т. е. при $\gamma=\gamma_{C R} ; T=T_{t r}=T_{C}$. В этой точке зависимость $\Delta S_{M}^{\max }$ от магнитного поля имеет вид [10]:

$$
\Delta S_{M}^{\max } \approx\left(2 \mu_{B} s H / k_{B} T_{c}\right)^{2 / 5}
$$

Сравнение численных значений величин приращения энтропии, описываемых формулами (8) и (9) для одних и тех же $s, H$ и $T_{C}$ показывает, что величина (9) почти на порядок больше, чем (8), т. е. максимальное значение изменения энтропии значительно возрастает для ферромагнетиков в окрестности именно магнитного фазового перехода I рода.

Этот вывод подтверждает и результат модельного расчета по формуле (4) для ферромагнетика $\mathrm{La}\left(\mathrm{Fe}_{0,88} \mathrm{Si}_{0,12}\right)_{13}$. Действительно, согласно экспериментальным данным работ $[11,12]$, для ферромагнетика $\mathrm{La}\left(\mathrm{Fe}_{0,88} \mathrm{Si}_{0,12}\right)_{13}$ наблюдалось: $N=6,07 \cdot 10^{22} \mathrm{~cm}^{-3} ; K=1,1 \cdot 10^{12}$ эрг $/ \mathrm{cm}^{3}$; $s=1 ; T_{C}=195 \mathrm{~K} ; \Theta=400$ К. С этими значениями физических величин, как следует из [10], $\gamma_{C R}=3,67 \cdot 10^{-13}$ эрг. Так как данный ферромагнетик испытывает фазовый переход I рода, то характеризующее его значение $\gamma$ получается больше, чем $\gamma_{C R}: \gamma=4,89 \cdot 10^{-13}$ эрг.

На рис. 1 и 2 показаны результаты расчета по формулам $(2,4)$ температурной зависимости изменения энтропии в магнитном поле при различных значениях отношения $\gamma / \gamma_{C R}$. Численные значения всех остальных физических величин (за исключением $\gamma$ ), которые входят в расчетные формулы, приняты такими же, как для ферромагнетика $\mathrm{La}\left(\mathrm{Fe}_{0,88} \mathrm{Si}_{0,12}\right)_{13}$. Как видно, максимальное значение изменения энтропии и пропорционального ей адиабатического изменения температуры наблюдается в точке Кюри. Из результатов расчета следует сильная зависимость величины МКЭ от значений постоянной магнитоупругого взаимодействия.

Вывод о том, что в большом числе магнитоупорядоченных соединений наивысшие значения МКЭ достигаются в образцах с магнитными фазовыми переходами I рода, подтверждают и результаты работы [7]. Причиной больших величин $\mathrm{MKЭ} \mathrm{в} \mathrm{RCo}_{2}$ является сильная зависимость намагниченности от температуры и магнитного поля в окрестности магнитного фазового перехода I рода, который имеет место в этих соединениях. Постепенное уменьшение величины МКЭ в ряду соединений $\mathrm{RCo}_{2}(\mathrm{R}=\mathrm{Er}, \mathrm{Ho}, \mathrm{Dy}, \mathrm{Tb})$ связано с ослаблением резкости перехода I рода в этом ряду и его исчезновением для $\mathrm{TbCo}_{2}$. 


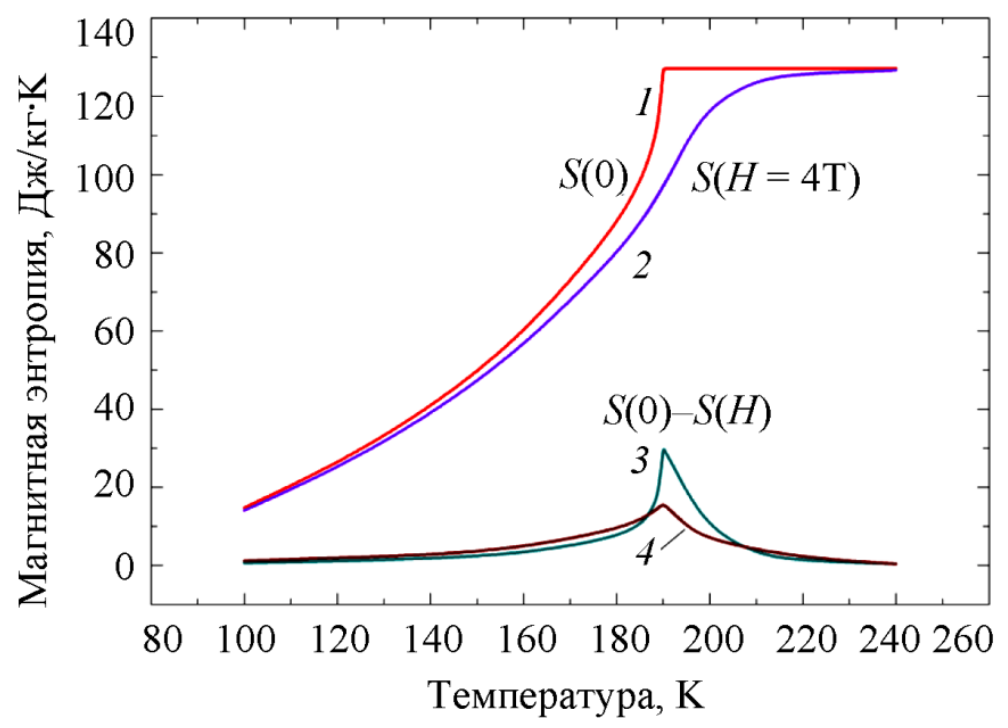

Рис. 1. Температурная зависимость магнитной энтропии при различных значениях магнитного поля $H=0$ и $H=4$ тесла. Расчет по формулам $(4,5)$. Кривые $1,2,3$ результат расчета для $\gamma / \gamma_{\text {кр }}=1$. Кривая $4-$ для $\gamma / \gamma_{\text {кр }}=0,67$

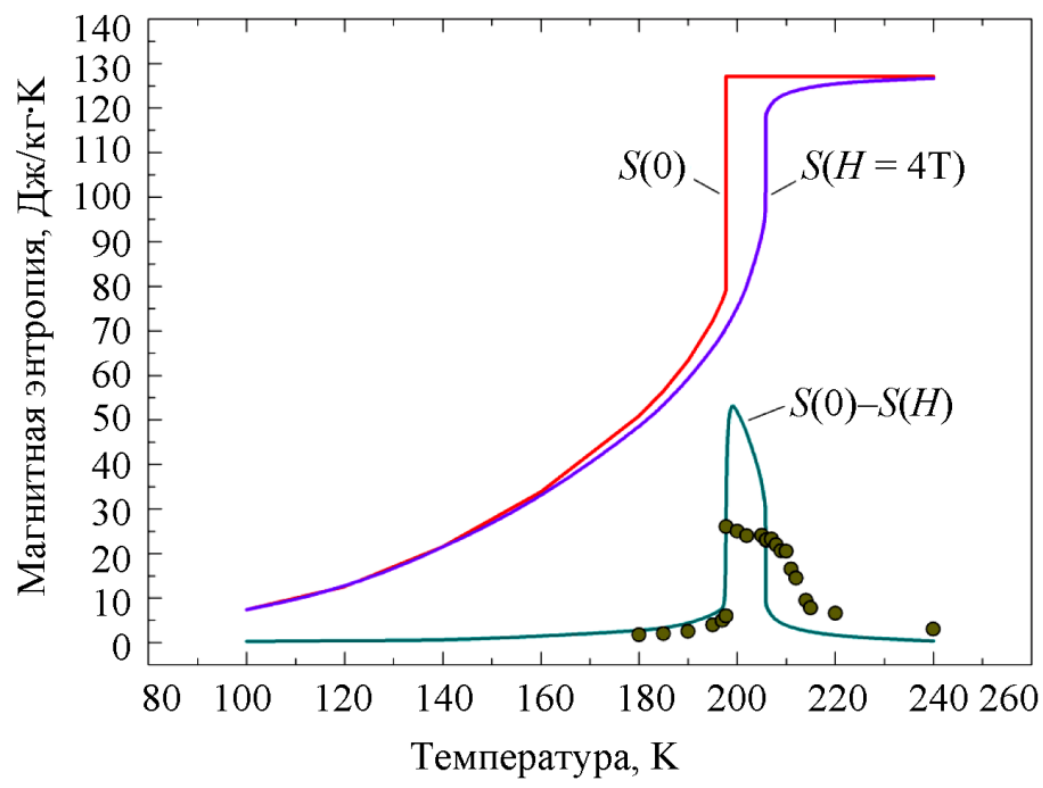

Рис. 2. Температурная зависимость магнитной энтропии при магнитном фазовом переходе

I рода для ферромагнетика $\mathrm{La}\left(\mathrm{Fe}_{0,88} \mathrm{Si}_{0,12}\right)_{13}$. Сплошные кривые - расчет по формулам в тексте статьи. Кружки - эксперимент из [11]

Увеличение абсолютных величин $\Delta S_{M}^{\max }$ и $\Delta T_{a d}^{\max }$ в ферромагнетиках с магнитными фазовыми переходами I рода, по сравнению с теми, которые имеются в точке Кюри при фазовых переходах II рода, сопровождается изменением характера температурной зависимости этих величин (рис. 1 и 2). Для ферромагнетиков с переходом II рода имеется один фазовый переход в точке Кюри $(H=0)$. В этом случае намагниченность при $T_{C}$ изменяется непрерывно, как при отсутствии магнитного поля, так и в магнитном поле. Соответственно температурная зависимость $\Delta S_{M}^{\max }(T)$ демонстрирует лишь один острый пик [9] (рис. 1).

Для ферромагнетиков с магнитным фазовым переходом I его проявления наблюдаются в виде скачков намагниченности при температуре $T_{C}(H=0)$, а также в точке $T_{C}(H \neq 0)$. Причем $T_{C}(H \neq 0)>T_{C}(H=0)$. Поэтому в температурной зависимости $\Delta S_{M}^{\max }(T)$ наблюдается 
П-образная кривая с двумя точками резкого изменения этой величины при указанных выше температурах (рис. 2). Величина скачка энтропии в точке $T_{C}(H=0)$ составляет $\approx 50 \mathrm{~J} / \mathrm{kg} \mathrm{K}$, что соответствует величине разрыва намагниченности $\Delta m \approx 0,7$. Экспериментальные данные подтверждают эти выводы. При сравнении рисунков 1 и 2 необходимо также обратить внимание на резкое возрастание численных значений $\Delta S_{M}^{\max }\left(T_{C}\right)$ при фазовых переходах I рода.

Из формулы для термодинамического потенциала (2) следует также выражение для решеточной части энтропии [5]:

$$
S_{F}=-N k_{B}\left[3 \ln \left(1-\exp \left(-\frac{\Theta}{T}\right)\right)-4 D\left(\frac{\Theta}{T}\right)\right] .
$$

С помощью формул (5) и (10) можно вычислить величину $\Delta T_{a d}^{\max }$. Она определяется из условия адиабатичности:

$$
S_{M}^{\max }(T, 0)+S_{F}(T, 0)=S_{M}^{\max }\left(T+\Delta T_{a d}^{\max }, H\right)+S_{F}\left(T+\Delta T_{a d}^{\max }, H\right) .
$$

Здесь предполагается, что электронный вклад в полную энтропию ферромагнетика мал.

Результат расчета адиабатического изменения температуры ферромагнетика $\mathrm{La}\left(\mathrm{Fe}_{0,88} \mathrm{Si}_{0,12}\right)_{13}$ при включении магнитного поля $H=4$ Т представлен на рис. 3 , он удовлетворительно согласуется с экспериментом.

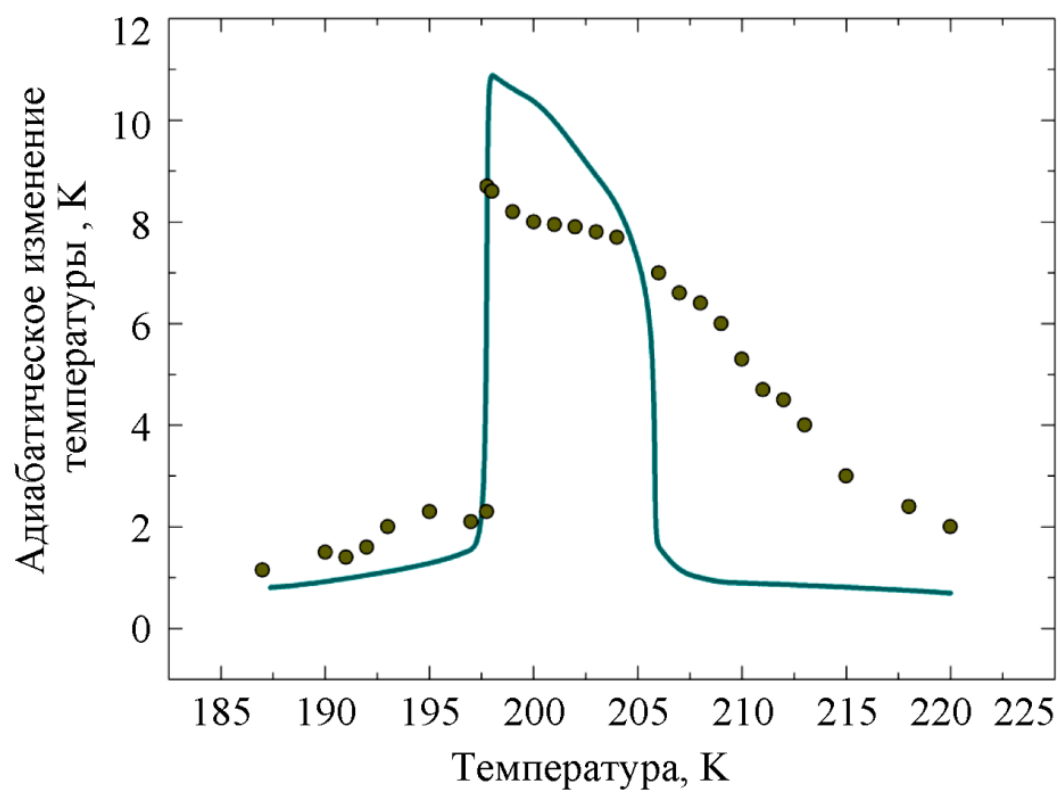

Рис. 3. Адиабатическое изменение температуры при включении магнитного поля 4Тл в зависимости от исходной температуры ферромагнетика $\mathrm{La}\left(\mathrm{Fe}_{0,88} \mathrm{Si}_{0,12}\right)_{13}$. Сплошная линия результат расчета по формуле (9). Кружки - экспериментальные данные работы [11]

В работе [8] также показано, что применение обменно-стрикционной модели и модели Киттеля позволяет количественно объяснить основные магнитные свойства (температурной зависимости намагниченности и относительного изменения объема, зависимости $T_{N}$ и $T_{C}$ от давления) антиферромагнитной и ферромагнитной фаз сплава Fe-Rh. B этой работе продемонстрировано, что предположение об отрицательной самопроизвольной объемной магнитострикции в антиферромагнитном состоянии и положительном - в ферромагнитной фазе приводит к теоретическим результатам, воспроизводящим экспериментальные данные. Причем наличие сильной зависимости обменных интегралов антиферромагнитной и ферромаг- 
нитной фаз сплава $\mathrm{Fe}-\mathrm{Rh}$ от объема (давления и температуры) являются важными моментами при анализе рекордного МКЭ этого сплава.

\section{4. Выводы}

Таким образом, наш анализ и экспериментальные данные свидетельствуют, что большая величина МКЭ наблюдается в том случае, когда при магнитном фазовом переходе объем немагнитной и магнитоупорядоченной фаз заметно различается. Так, для соединений $\left(\mathrm{La}\left(\mathrm{Fe}_{\mathrm{x}} \mathrm{Si}_{1-\mathrm{x}}\right)_{13} ; \mathrm{RCo}_{2}\right.$ и $\mathrm{Fe}-\mathrm{Rh}$ спонтанная объемная магнитострикция $\omega \sim 10^{-2}$ и $\gamma \approx 10^{-12}$ эрг $\left(10^{-19}\right.$ дж) [5-8]. Эти численные значения $\omega$ и $\gamma$ можно принять за ориентир при поиске новых соединений с большим МКЭ.

Анализ особенностей зависимости $\Delta S_{M}^{\max }$ от магнитного поля для ферромагнетиков с фазовыми переходами первого и II рода, выполненный в рамках предлагаемого обобщенного феноменологического подхода, показывает, что рекордные значения $\Delta S_{M}^{\max }$ порядка (20-40) J/kg K и значения $\Delta T_{a d}^{\max }$ порядка $10 \mathrm{~K}$ требуют использования соединений с магнитными моментами 5-10 $\mu_{\mathrm{B}}$ на атом и $T_{C}$ примерно $300 \mathrm{~K}$. При этом для магнетиков с фазовыми переходами I рода величина скачка намагниченности должна быть по-

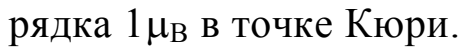

\section{Благодарность}

Работа выполнена с использованием УНУ «НМК ИФМ» в рамках Госзадания ИФМ УрО РАН (тема «ПОТОК») при поддержке гранта № 18-10-2-22 программы фундаментальньхх исследований УрО РАН.

\section{Литература}

1. Magnetocaloric effect: From materials research to refrigeration device / V. Franco, J. S. Blázquez, J. J. Ipus, J. Y. Law, L. M. Moreno-Ramírez, A. Conde // Progress in Materials Science. - 2018. - Vol. 93, pp. 112-232. - DOI: 10.1016/j.pmatsci.2017.10.005.

2. Description and performance of a near-room temperature magnetic refrigerator / C. Zimm, A. Jastrab, A. Sternberg, V. Pecharsky, K. Gschneidner, M. Osborne, I. Anderson // Advances in cryogenic engineering book series (ACRE, vol. 43) / ed. by P. Kittel. - Boston : Springer, MA, 1998. - Vol. 43. - P. 1759-1766. - DOI: 10.1007/978-1-4757-9047-4_222. - Online ISBN: 978-14757-9047-4.

3. Bean C. P., Rotbell D. C. Magnetic disorder as first-order phase transition // Phys. Rev. 1962. - Vol. 126. - P. 104-115. - DOI: 10.1103/PhysRev.126.104.

4. Kittel C. Model of exchange-inversion magnetization // Phys. Rev. - 1960. - Vol. 120. P. 335-342. - DOI: 10.1103/PhysRev.120.335.

5. Valiev E. Z., Kazantsev V. A. Magnetocaloric effect in $\mathrm{La}\left(\mathrm{Fe}_{x} \mathrm{Si}_{1-\mathrm{x}}\right)_{13}$ ferromagnets // Physics of Metals and Metallography. - 2011. - Vol. 113, no. 6. - P. 1000-1005. DOI: $10.1134 / \mathrm{S} 1063776111150118$.

6. Valiev E.Z. Isotropic Magnetoelastic Interaction in Two-Sublattice Ferriand Antiferromagnets: Mean-Field Approximation for the Heisenberg Model // Physics of Metals and Metallography. - 2003. - Vol. 96, no. 2. - P.121-127.

7. Valiev E. Z. Entropy and Magnetocaloric Effect in Ferrimagnets $\mathrm{RCo}_{2} / /$ Physics of Metals and Metallography. - 2017. - Vol. 124, no. 6. - P. 968-974. - DOI: 10.1134/S1063776117060048.

8. Application of the exchange-striction model for the calculation of the FeRh alloys magnetic properties / E. Valiev, R. Gimaev, V. Zverev, K. Kamilov, A. Pyatakov, B. Kovalev, A. Tishin // Intermetallics. - 2019. - Vol. 108. - P. 81-86. - DOI: 10.1016/j.intermet.2019.02.015. 
9. Valiev E. Z. Entropy and Magnetocaloric Effect in Ferromagnets and Antiferromagnets // Physics of Metals and Metallography. - 2007. - Vol. 104, no. 1. - P. 8-12. DOI: $10.1134 / \mathrm{S} 0031918 X 07070022$.

10. Valiev E. Z. Entropy and magnetocaloric effects in ferromagnets undergoing first- and second-order magnetic phase transitions // JETP. - 2009. - Vol. 108, no. 2. - P. 279-285. DOI: $10.1134 /$ S1063776109020101.

11. Fujita A., Akamatsu Y., Fukamishi K. Itinerant-electron metamagnetic transition in $\mathrm{La}\left(\mathrm{Fe}_{\mathrm{x}} \mathrm{Si}_{1-\mathrm{x}}\right)_{13}$ intermetallic compounds // J. Appl. Phys. - 1999. - Vol. 85. - P. 4756-4758. - DOI: 10.1063/1.370471.

12. Itinerant-electron metamagnetic transition and large magnetocaloric effect in $\mathrm{La}\left(\mathrm{Fe}_{\mathrm{x}} \mathrm{Si}_{1-\mathrm{x}}\right)_{13}$ compounds and their hydrides / A. Fujita, S. Fujieda, Y. Hasegava, K. Fukamishi // Phys. Rev. B. 2003. - Vol. 67, no. 10. - P. 104416. - DOI: 10.1103/PhysRevB.67.104416. 